\title{
EL IMPACTO DE LAS REPRESENTACIONES SOCIALES EN LA PSICOLOGÍA DE LOS CONOCIMIENTOS SOCIALES: PROBLEMAS Y PERSPECTIVAS
}

\author{
JOSÉ ANTONIO CASTORINA \\ Instituto de Investigaciones en Ciencias de la Educación, Universidad de Buenos Aires y \\ Consejo Nacional de Investigaciones Científicas y Técnicas \\ ctono@netizen.com.ar
}

\begin{abstract}
RESUMO
Los estudios psicológicos sobre la formación de nociones sociales de los niños se han llevado a cabo en los términos de una actividad intelectual exclusivamente individual y en una secuencia temporal. Se muestran las dificultades de este enfoque, como por ejemplo, que no explican la perduración de nociones "personalizadas" de la historia durante el desarrollo, más allá del avance propiamente conceptual. Se propone utilizar a la teoría de las representaciones sociales, que considera a al niño como un actor social y cuyo foco está en los valores, que no pueden ser organizados en una secuencia lógica. Luego, se examinan los problemas que involucra utilizar las representaciones sociales en la psicología del desarrollo: si su definición es aceptable, si corresponde al orden simbólico y no a una actividad individual; si son irracionales o tienen otra lógica que los conceptos individuales. Finalmente, se aclaran cuáles son las condiciones epistémicas mínimas para establecer un diálogo entre algunos programas de investigación en psicología del conocimiento y la psicología de las representaciones sociales.

REPRESENTACIONES SOCIALES - VALORES MORALES - DESARROLLO COGNOSCITIVO
\end{abstract}

\section{ABSTRACT}

THE IMPACT OF SOCIAL REPRESENTATIONS ON THE PSYCHOLOGY OF SOCIAL KNOWLEDGE: ISSUES AND PERSPECTIVES. Psychological studies on the formation of social notions by children have been conducted in terms of an exclusively individual intellectual activity in a time sequence. We show the difficulties of this approach, which does not, for example, explain the persistence of "personalized" notions of history during development, besides actual conceptual advancement. We propose to use the theory of social representations, which considers the child as a social actor and focuses on values, which may not be organized in a logical sequence. We then examine the issues posed by using social representations in developmental psychology: whether its definition

Subsidió la escritura del artículo la Agencia Nacional de Promoción Científica y Tecnológica ANPCYT (n. 2006-34778). 
is acceptable, whether it corresponds to the symbolic order and not to an individual activity; whether it is irrational or has a logic other than individual concepts. We finally shed light on the minimum epistemic conditions for establishing a dialogue between some investigation programs in knowledge psychology and the psychology of social representations.

SOCIAL REPRESENTATIONS - MORAL VALUES - COGNITIVE DEVELOPMENT

La teoría de las representaciones sociales (en adelante RS) ha estado vinculada en su origen con dos pensadores mayores de la psicología del desarrollo. Moscovici (2003), por un lado, ha reconocido explícitamente su deuda con Vigotsky, básicamente por su tesis de la socialización de la vida psicológica y el rol jugado por la cultura en la constitución de los fenómenos psíquicos superiores. Por otro lado, la preocupación de Piaget en la transformación de los conocimientos y sobre todo su tesis constructivista de la interacción del objeto con el sujeto influyeron decisivamente en el fundador de la psicología de las RS (Castorina, Barreiro, Clemente, 2005).

Ahora bien, el estado actual de las investigaciones y de los debates teóricos nos colocan en la situación recíproca: las RS pueden contribuir a resolver ciertos problemas que se plantean en distintos programas de investigación en la psicología del desarrollo. Así, la psicología de Vigotsky ha explicado la génesis de los procesos psíquicos superiores por la apropiación de los instrumentos culturales, pero ha mostrado algunas insuficiencias. Particularmente, su interpretación de estos últimos ha resultado excesivamente simplificadora y homogeneizante. En este sentido, la utilización de las RS promete ser pertinente para diversificar el concepto de cultura, introduciendo los valores grupales en la homogeneidad de la concepción formulada por Vigotsky, incluso promoviendo la intervención de las identidades sociales como una mediación entre aquellos instrumentos y los procesos intraindividuales (Duveen, 1998; Van der Veer, 1996; Castorina et al., 2007).

Vamos a ocuparnos en este trabajo, sin embargo, de los estudios sobre el desarrollo de los conocimientos sociales en los niños, inspirados en el programa cognitivista y principalmente en el programa piagetiano. Básicamente, estamos ante explicaciones basadas en la elaboración de conceptualizaciones cada vez más "avanzadas" en los conocimientos sobre la historia, la autoridad política o escolar, las nociones económicas, o aún morales. En cualquier caso, los psicólogos investigan la elaboración de representaciones sobre el mundo 
social en los términos de una actividad exclusivamente individual de construcción conceptual o elaboración de la información; más aún, el mundo social es considerado por los investigadores como enteramente exterior a los sujetos de investigación.

Tales estudios presentan algunas dificultades: en primer lugar, resulta sospechoso que se examinen los conocimientos sociales sin la intervención relevante de las prácticas sociales. En esta perspectiva, la cultura y las condiciones sociales intervienen solo en tanto frenan o aceleran un desarrollo cognoscitivo que es autónomo. Además, dichos estudios no explican la perduración en la historia individual de ciertas ideas que resisten a su modificación durante el desarrollo de los conceptos y representaciones propiamente cognitivas, incluso durante los procesos de enseñanza y aprendizaje. Incluso, tales indagaciones utilizan como referencia para estudiar la formación de las ideas infantiles a los conocimientos del "sentido común" del adulto, sin tomar en cuenta los conocimientos más avanzados en las ciencias sociales contemporáneas, en abierta contradicción con lo que Piaget había propuesto.

Solo mostramos aquí un par de ejemplos de lo insatisfactorio que resulta explicar el conocimiento social apelando solamente a las representaciones conceptuales: la persistente "personalización" en la caracterización infantil, que perdura durante toda la vida de las personas, de la autoridad política y de los fenómenos históricos, que perdura durante toda la vida de las pessoas. Los niños y adolescentes tienden a considerar que la autoridad presidencial concentra el poder, se ocupa de hacer el bien, no tiene límites a su accionar, salvo morales; la interpretación de la historia centrada en las intenciones individuales, por ejemplo, que la conquista de América se debe a las ambiciones y rasgos personales de los actores históricos, en niños y jóvenes. Además de la personalización de la vida social, podemos mencionar la creencia ampliamente difundida en los conocimientos históricos "de que la nación existe desde siempre... es prácticamente eterna, algo que no se puede borrar de un golpe... el territorio argentino existe desde la aparición del hombre... desde antes de la aparición del hombre..." (Carretero, Kriger, 2006).

El hecho es que la "personalización" y la noción sustancialista de "nación" siguen apareciendo en las indagaciones, incluso más allá del avance conceptual de los sujetos y muestran una resistencia a su modificación "argumentativa". Las características de las respuestas de los sujetos - dentro y fuera de la sala 
de clase - indican la existencia de rasgos que parecen irreductibles al proceso de génesis de los sistemas conceptuales, tales como dificultades en las abstracciones o en la diferenciación e integración de las ideas.

¿La teoría de las RS puede aspirar a resolver algunas de las dificultades señaladasi

En primer lugar, las RS se distinguen en aspectos relevantes del conocimiento propiamente conceptual y de las representaciones estudiadas por los psicólogos del conocimiento. Estas son elaboradas a través de la comunicación, de modo que existen dentro de una estructura de lazos asociativos de significación. Y sobre todo, son inherentemente evaluativas y toman forma a través del discurso social, no estando limitadas por los cánones de la lógica argumental de los conceptos. En breve, su foco está en el mundo de los valores en adición al mundo de los hechos, lo que introduce una dificultad en la interpretación del desarrollo intelectual: los valores no pueden ser organizados en la secuencia lógica que podría proveer, justamente, la estructura de un estudio de desarrollo.

Por lo dicho, a la perspectiva del niño como un constructor individual del conocimiento social se le contrapondría la tesis del niño como un actor social (Duveen, de la Rosa, 1992). Así, se verifica la variación de las expectativas infantiles sobre el comportamiento de los maestros en una situación escolar en función de su pertenencia grupal (Emler, Ohana, Moscovici, 1987); también se encuentran niños entre 3 y 12 años que consideran a los salarios ganados por las mujeres menores que los pagados a los varones, sin variaciones con la edad aunque sí con la pertenencia de género (Duveen, 1989). De ahí que las evaluaciones infantiles del comportamiento de los maestros o la economía no pueden ser situadas simplemente en una secuencia lineal de "inexpertos" a "expertos" o de pensamiento "prelógico" a pensamiento "lógico". O, por lo menos, hay aquí un serio problema de compatibilidad entre programas de investigación, como vamos a discutir.

Ahora bien, iqué condiciones deben reunir las RS para ser utilizadas por un psicólogo del desarrollo? o iqué dificultades frecuentemente asociadas con las RS deben ser superadas para su incorporación a la psicología del desarrollo? Más aún, ies preciso revisar las tesis de los programas de investigación del desarrollo para permitirlo? Principalmente, isobre qué base discutir la posibilidad de un intercambio entre las disciplinas? 


\section{EL ANÁLISIS CONCEPTUAL}

Las cuestiones que hemos formulado son metateóricas, ya que apuntan a caracterizar los conceptos que forman parte de un campo disciplinario y establecer la posibilidad de ser incluidos eventualmente en otro campo. Mientras una teoría se elabora para dar cuenta de la recurrencia de ciertos datos y la investigación empírica produce prueba empírica para las hipótesis teóricas, el análisis conceptual se ocupa de la inteligibilidad y la consistencia de los conceptos elaborados. Sin duda, hay una interdependencia entre este análisis y las dimensiones teóricas y empírica de la investigación psicológica (Machado, Lourenço, Silva, 2000).

Dicho análisis se ocupa de las categorías que emplean los investigadores para los conocimientos sociales a los fines de establecer la vaguedad o la precisión de su definición, las condiciones que han promovido su elaboración, así como los problemas de articulación teórica que derivan de su coordinabilidad con las tesis de otro campo disciplinario. En este caso, nos preguntamos si es aceptable integrar a la psicología del conocimiento individual el concepto de RS que pertenece a otro campo disciplinario, el que recorta de modo muy diferente los problemas y utiliza sus procedimientos específicos de indagación. Otra tarea de este tipo de investigación es explicitar los presupuestos filosóficos que han intervenido en las decisiones metodológicas y en los modelos explicativos que emplean.

Estas reflexiones pertenecen a la epistemología "interna" de la psicología del desarrollo y de la psicología social, pero "mientras se llevan a cabo" las investigaciones teóricas y empíricas, a las que están estrechamente asociadas. No se trata de examinar el corpus teórico "ya terminado" de una disciplina, como lo hacen con frecuencia los filósofos, perdiendo de vista la historicidad de los conceptos y de los objetos de la psicología y por tanto las condiciones que los hicieron posibles. Por el contrario, para pensar los problemas que plantea la noción de RS para la psicología del desarrollo hay que reconocer los entrecruzamientos, las influencias y los escollos que provienen de la conformación y la actualidad de los dos campos disciplinarios.

Se trata de una tarea para los psicólogos de las disciplinas, ya que ellos pueden reconstruir los problemas que suscitaron la creación o el empleo de los conceptos, tomar conciencia de sus dificultades y proponer modificaciones en el corpus teórico en el que se inserta o aún abandonarlo. Vamos a exami- 
nar la pertinencia de las RS para nuestra disciplina, la psicología del desarrollo de los conocimientos sociales, "situándonos desde el espacio de sus propios problemas y sus compromisos teóricos".

Ahora bien, la utilización de las RS debe sortear, en primer lugar, la desconfianza de muchos psicólogos ante ciertas supuestas dificultades conceptuales de la categoría: si su definición es aceptable aunque se formula aún con cierta vaguedad; si está debidamente establecido que la "representación" corresponde al orden simbólico y no a una actividad individual; si se puede rechazar la tesis que las RS "carecen de lógica". Y sobre todo, hay que aclarar cuáles son las condiciones epistémicas mínimas que deben cumplirse para que los programas de investigación puedan entrecruzarse o intercambiar sus producciones.

En segundo lugar, nos interesa especialmente el programa constructivista al que pertenecen nuestras propias investigaciones en psicología de los conocimientos sociales (Lenzi, Castorina, 2000; Horn, Castorina, 2007; Barreiro, Castorina, 2006). Respecto de dicho programa, proponemos explicitar y aún modificar algunos aspectos de su núcleo para compatibilizarlo con la teoría de las RS (en adelante TRS).

\section{LA DEFINICIÓN DE RS}

Nos enfrentamos ante la primera cuestión propiamente epistemológica: los esfuerzos de los psicólogos ihan logrado producir una definición suficientemente rigurosa de las RS para ser tomada en cuenta por los psicólogos del desarrollo? En diferentes ámbitos de la psicología del desarrollo (Jahoda, 1988; Delval, 1994) se las ha cuestionado debido al carácter insatisfactorio de su definición, por ser imprecisa y carecer de unicidad, lo que la vuelve inapropiada para ser utilizada en la psicología de los conocimientos.

Ante todo, hay que decir que no disponemos de una definición apriori de las RS, que alcance la precisión y rigor que los filósofos suelen exigirle para ser satisfactoria en una teoría. A poco de ser elaborada en sus líneas más generales por Moscovici, la RS fue cuestionada por diversas razones y desde diferentes perspectivas. Por una parte, se le ha reprochado que su definición es imprecisa, de modo tal que no es posible distinguirla de otras categorías sociales. Se le ha atribuido además, una gran vaguedad y ambigüedad en la formulación de la teoría (Jahoda, 1988; Smedslund, 1998; Delval, 1994). 
Sin duda, Moscovici ( 1988) eligió una cierta vaguedad para el concepto, lo que se entiende porque la historia de la psicología social ha mostrado que cada vez que la precisión de las definiciones ha sido buscada obsesivamente se ha pagado el costo del compromiso con el fenómeno social. Esto es, los conceptos suelen adquirir rigor formal en la caracterización, como en el caso de las actitudes, pero pierden el sentido del fenómeno social. Más aún, la claridad formal lleva por su parte a la vaguedad de aquello a lo que pretende referirse.

Dicha vaguedad no es el centro del concepto o no es su nota mas destacada, ya que no ha bloqueado la formulación de investigaciones de relevancia empírica, y ha permitido iluminar ciertas zonas de fenómenos. Más aún, ha dado lugar a explicaciones de la sociogénesis de las creencias sociales que han resultado esclarecedoras. En síntesis, puede decirse que las RS tienen un mínimo de precisión, el que ha posibilitado el despliegue de un extenso cuerpo de investigaciones empíricas (Duveen, 1998).

Por otra parte, la exigencia de una definición estrictamente rigurosa de las RS revela una incomprensión sobre su estatuto teórico: en cualquier ciencia social aquella caracterización no corresponde al punto de partida sino al punto de llegada de una laboriosa tarea. De hecho, nos parece encontrar en muchas investigaciones de la TRS una continuada interacción entre el conocimiento teórico y los datos producidos que contribuye a refinar las notas del concepto; y sobre todo, la variedad de definiciones elaboradas por los discípulos de Moscovici no indica un déficit inherente a dicha producción teórica, sino niveles de elaboración vinculados con diferentes problemas de investigación.

\section{EL “DEMONIO'” DE LA REPRESENTACIÓN COGNITIVA}

En segundo lugar, se trata de establecer si la TRS ha tomado una efectiva distancia de las "representaciones" cognitivas, asociadas a la psicología de la información. Como es sabido, los psicólogos discursivos (Potter, Edwards, 1999) han señalado que a pesar de la intención de Moscovici en diferenciarse de la representación cognitiva, las RS la prolongan: provienen de la actividad mental de los individuos y están disociadas del mundo externo. Si no se puede

I. El témino "demonio" está puesto porque las críticas persistentes a las RS se apoyan em lo que no se las puede distinguir de las representaciones individuales. 
establecer diferencias significativas entre las representaciones, no habría una genuina contribución de las RS a la psicología del desarrollo. Es decir, nos estaríamos moviendo dentro del mismo espacio intelectual y no se ve la originalidad de la contribución de las RS a la psicología del desarrollo.

Una mirada a la historia de los significados del término "representación" en la cultura europea se opone a la identificación propuesta por los críticos (Markova, 2003). En la tradición intelectual francesa de la que proviene el concepto de representación colectiva de Durkheim, el término tuvo una significación polisémica. Es decir, junto al sentido mental de "figurar al mundo", el propio Dictionnaire Robert le atribuye un sesgo claramente dialógico. En la significación del término se incluye una relación constitutiva con algún otro, "... un objeto ausente se hace significativo para otros por medio de una imagen o signo o por el discurso" (idem, p. I19).

En el siglo XVIII, el término tiene dos sentidos: por un lado, la representación significa "... ver el objeto ausente", y en el sentido político o jurídico "... ocupar el lugar de alguien, tener en mano su autoridad", "se dice en Palacio de la exhibición de algo, lo que significa también comparecer en persona y exhibir las cosas" (Chartier, 1995). Sin duda, la cultura francesa no reduce la representación a sus aspectos cognitivo individuales.

En cambio, en la lengua inglesa se connota una imagen o una reproducción especular y estática del mundo como parte del pensamiento moderno, hasta la teoría del procesamiento: la separación de las formas mentales respecto de la vida social.

En la tradición francesa, las RS se caracterizan por restituir simbólicamente al objeto para un sujeto grupal o social y en la comunicación social. El "representar algo para alguien" (Moscovici, 1986) pone de relieve que están orientadas intrínsecamente a la comunicación con otro.

Además, la TRS cuestiona la disociación de las representaciones con respecto al mudo exterior y a la vida social, así como la elaboración individual de informaciones provenientes de un mundo exterior ya dado, característica de la psicología computacional. En cambio, las RS provienen de una construcción en la que se comprometen los individuos en tanto agentes sociales, como miembros de una clase o un grupo e involucran una actividad simbólica que surge de la interrelación entre el yo, el otro y el mundo, produciendo significados que crean "la realidad" para los grupos (Jovchelovitch, 2006). 
En síntesis, a diferencia de las tesis de la psicología cognitiva, el predicado "cognitivo" se aplica solamente a los actos individuales que son a la vez sociales, que construyen su saber "con otros", expresan el punto de vista del grupo, y se modifican en la interacción comunicativa.

\section{LA LÓGICA NATURAL DE LAS RS}

Para un psicólogo que estudia la formación de argumentos infantiles acerca de los fenómenos sociales sería impensable que la categoría que se pretende incorporar sea ilógica o solo ofrezca inconsistencia en sus significados. ¿Cuál sería la defensa de la TRS ante el reproche de irracionalidad?

La noción de representación en la tradición de la TRS, como hemos dicho, no es la reproducción de un objeto, sino una elaboración de significados que lo estructuran, pero a la vez su propia articulación involucra un plus con su articulación. Así, si evocamos los mecanismos de condensación y desplazamiento postulados por Freud, los símbolos pueden representar un entramado de significaciones, cuyos elementos pueden ser lógicamente contradictorios, aunque pueden coexistir en las representaciones psíquicas, lo que indica otra lógica respecto a la que rige el conocimiento del mundo (Duveen, 1998).

Por su parte, las RS tienen un núcleo "figurativo" alrededor del cual se puede hablar de la coalescencia de diversos significados, que entre si no tienen una estricta consistencia. Más aún, las RS no solo estructuran significativamente las prácticas sociales, sino que ocultan algunos de sus aspectos relevantes. Son a la vez un modo de comprender algo y un modo de no comprender otros aspectos: la "personalización" de la vida democrática afirmada por los niños refiere a relaciones directas por delegación entre presidente y gobernados, pero rechaza la representación indirecta de diputados y senadores, valorando negativamente su rol político (Lautier, 1997). Aquí se resaltan algunos rasgos figurativos y familiares de la política, pero se ocultan otros, referidos a la institucionalidad y al sentido del sistema de representación política. El sentido común "interpreta el fenómeno" y a la vez "oculta y oscurece algo de él".

Ahora bien, esos significados se inscriben en un entramado lógico peculiar por la combinación de los significados, en algún sentido semejantes a la participación de que hablaba Levi-Bruhl o al pensamiento transductivo que conectaba lo particular a lo particular sin recurrir a la universalidad ni a la necesidad lógica, 
en el primer Piaget (1940). La articulación lógica de las representaciones conceptuales no se contrapone con la ausencia de lógica de las RS, sino con otro tipo de implicaciones, otras articulaciones de los significados.

Más aún, Jean Blaise Grize (1986) ha mostrado una lógica específica en las RS, procesos de pensamiento que conjugan forma y contenido a través de la conformación de los objetos semióticos, y durante los discursos dirigidos a un interlocutor. En esta "lógica natural" los aspectos hipotético deductivos de la "lógica científica" e incluso de la "lógica operatoria" de Piaget se esfuman. Los individuos apelan durante las relaciones discursivas a las "causas" de los hechos consumados, a la autoridad de quien habla o de quien se habla, a las comparaciones con analogías "salvajes", a un cierto bricolage de las RS en tanto "referencias para la acción" (Lautier, 2006).

Aquella rígida contraposición entre la marcha del conocimiento hacia la racionalidad y el carácter irracional de todo proceso alternativo, que subyace a una buena parte de la psicología cognitiva del desarrollo, supone la tesis de la unicidad del desarrollo del pensamiento y de un criterio trascendental que decide sobre otras formas de pensamiento. Estos rasgos son característicos del proyecto intelectual de la modernidad y ya no son aceptables (Jovchelovich, 2006).

\section{LAS RELACIONES ENTRE LOS PROGRAMAS}

Hemos tratado de ofrecer un análisis de algunos aspectos centrales de la TRS que son relevantes para repensar a la psicología del desarrollo, ahora tenemos que examinar si los programa de investigación de esta última admiten tal categoría, o bien si hay que introducir cambios en su estructura teórica. Según lo visto, estamos ante dos versiones claramente diferentes de "representación", una que la sitúa en el interior de la vida mental (en la versión computacional) y la otra en las interacciones sociales; en un caso se trata únicamente de la construcción del pensamiento individual, en el otro interesa primordialmente "la sociedad pensante"; los psicólogos del desarrollo se ocupan de la conformación de sistemas de pensamiento articulados con una lógica estricta y la TRS exhibe más bien una lógica informal.

Justamente, las diferencias apuntadas son la clave para discutir una eventual colaboración entre las disciplinas ¿Cuál es la relación epistémica más 
sustentable entre los programas de la psicología del desarrollo y la TRS? iSe puede hablar de compatibilidad, incompatibilidad o inconmensurabilidad?

Muchos investigadores del desarrollo han decidido, explícita o implícitamente, ignorar simplemente a la TRS, sin siquiera preguntarse por las condiciones sociales de las ideas de los niños. Otra posición podría ser la celebración de la inconmensurabilidad "fuerte" entre el modo paradigmático y el modo narrativo o comprensivo de hacer psicología, siguiendo la perspectiva relativista propuesta por Bruner ( 1 997). Esto es, podría destacarse la diversidad de los sistemas teóricos en que se definen los conceptos, así como la inexistencia de situaciones empíricas "neutrales" que permitieran decidir sobre la validez de los programas. Vistas así las cosas, los términos de la TRS al ser comparados con la psicología del desarrollo no se pueden comprender ni traducir a sus términos (como el "representación" en la psicología computacional respecto de las RS).

En esta perspectiva, la distancia entre los programas sería insalvable porque sus hipótesis varían sustancialmente de acuerdo a la diferente centralidad de los conceptos de representación en las investigaciones. Esta es la posición de algunos psicólogos sociales. Así, Wagner ( 1992 ) ha sostenido que en los estudios de conocimiento social los conceptos explicativos de una teoría pertenecen a un espacio de problemas que los abarca. Así, los conceptos de la "social cognition" quedarían localizados dentro de un nivel de análisis intraindividual, de modo tal que las relaciones sociales quedarían afuera de consideración o serían explicadas reductivamente. Por el contrario, desde el desarrollo de la TRS se ha cambiado "el espacio de explicación del individuo hacia lo social" (p. I3). Se reconoce, entonces, una estructura integrativa, que abre al individuo para situarlo en la cultura y la sociedad, superando sus limites corporales. Según esta perspectiva, se puede evaluar los rasgos de cada programa según el espacio explicativo de base, en contra de comparaciones puntuales de ideas, conceptos y métodos de investigación. Aunque no lo afirma taxativamente, Wagner parece pensar que el estudio psicológico de las construcciones individuales presenta diferencias insalvables con la TRS, lo que imposibilita un intercambio fértil de conceptos y métodos.

Por su parte, Emler, Ohana, Dickinson (2003) consideran directamente incompatibles a los programas en cuestión: considerar a los niños como creadores de problemas y como creadores de respuestas se contrapone decididamente a postular que los problemas ya están planteados y resueltos 
en la sociedad (las RS). En otras palabras, afirmar que los sujetos se apropian en la comunicación social de las soluciones sociales ya dadas es inconsistente con la tesis constructivista que los sujetos "las construyen" individualmente. Los autores no subrayan la distancia entre los enfoques sino su contraste, ya que no hay indicadores empíricos de cualquier actividad reconstructiva por parte de los niños. En cambio, hay elementos de juicio para pensar que ellos reiteran la creencia de su grupo social. Por lo tanto, optan por el programa de la TRS, centrado en el estudio de los conocimientos sociales como determinaciones que van desde las RS hacia las creencias infantiles.

Por nuestra parte, preferimos utilizar la categoría de "marco epistémico" (en adelante ME) para el análisis de las relaciones entre los programas, que resulta más abarcativa respecto del "espacio de problemas" propuesto por Wagner, al incluir aspectos filosóficos de orden ontológico y epistemológico con su trasfondo social. Para Piaget y García (1982), un ME es una concepción del mundo que expresa relaciones sociales y culturales, y constituye el sentido común de los investigadores en un momento histórico, orientando la actividad científica. Su intervención en las investigaciones consiste en posibilitar el planteo de ciertos problemas en su campo, a la vez que hace "invisibles" a otros, orientando la investigación en una sola perspectiva metodológica o bien dando lugar a su diversidad.

Precisamente, una tarea relevante de la crítica epistemológica "interna" de la psicología del desarrollo es hacer explícitos a los presupuestos a los fines de evaluar sus efectos en la investigación. En este sentido, evocamos el ME de la escisión, propio del pensamiento moderno (Taylor, 1995) que separa ontológicamente la representación y el mundo, la mente del cuerpo, o el individuo de la sociedad; y además disocia epistemológicamente la observación con respecto de la teoría, y en ocasiones promueve explicaciones causales lineales de la adquisición de las ideas.

Insistimos, más que una teoría filosófica explícita, es una estrategia intelectual que al integrarse a la vida cultural circuló en los diversos ámbitos académicos y fue asumida de un modo más o menos implícito por buena parte de los psicólogos hasta hoy (Overton, 1998; Castorina, 2002). Muy especialmente, la disociación del individuo de la sociedad se ha impuesto ominosamente a los psicólogos y a otros científicos sociales por ser una consecuencia histórica del proceso civilizatorio (Elias, 1989). El homo clausus, resultado del proceso 
de individuación, hace casi imposible al investigador el distanciamiento que le permita situarse en un sistema de interrelaciones de conocimientos sociales, en la psicología cognitiva y en los estudios piagetianos clásicos sobre conocimientos sociales.

Según lo dicho, la "personalización" de la vida social e histórica, el carácter benefactor de la autoridad política o la interpretación esencialista de la nación, se explican en los estudios de psicología del desarrollo como resultado del proceso constructivo realizado por un sujeto irremediablemente solitario, y situado en una posición de exterioridad respecto de los hechos sociales. Tales rasgos del conocimiento social dependen del egocentrismo intelectual en el programa piagetiano o de la proyección de creencias mentalistas innatas sobre los fenómenos sociales, en el programa cognitivo.

Incluso, el ME de la escisión presidió no solo a la psicología del desarrollo que hemos mencionado, sino también los trabajos de aquellos investigadores de la TRS que negaron toda actividad intelectual a los individuos, reconociendo como única actividad la que ocurre en la participación de los niños en la comunicación de las RS (Emler, Ohana, Dickinson, 2003). Es decir proponiendo una disociación - que recuerda a Durkheim - entre individuo y sociedad. De este modo, la pertenencia social de los individuos determina unívocamente sus creencias, las que se interpretan como variables dependientes, en el espíritu de las explicaciones causales (Wagner, 1992).

Si nos atenemos al ME de la escisión, propio de estos psicólogos sociales y de los psicólogos del desarrollo antes comentados, no hay duda de que son incompatibles, porque se recurre a una explicación del conocimiento social por procesos puramente individuales que producen ideas en un orden secuencial y universal o bien a una determinación causal desde el grupo de pertenencia hacia las creencias individuales.

\section{UNA PSICOLOGÍA CONSTRUCTIVISTA COMPATIBLE CON LA TRS}

Afirmar que los programas de la psicología del desarrollo son incompatibles o inconmensurables con la TRS ies todo lo que puede decirse acerca de sus relaciones? Nosotros vamos a defender su compatibilidad en base a la tesis de un mismo ME relacional que preside las investigaciones constructivistas en la psicología del desarrollo y las que despliegan el núcleo de la TRS. 
Las obras de Moscovici (1984) y de Jodelet (1996), Duveen (200 I), Markova (2003) o Jovchelovitch (2006) han situado a las RS en el ME relacional o dialéctico, rechazando cualquier dualismo entre individuo y sociedad, sea el cognitivismo psicológico o la sociología de Durkheim. Claramente, se ha mostrado que la producción simbólica se da en las interrelaciones entre sujeto individual, el otro y el mundo-objeto, de modo tal que una RS emerge en un sistema de acción y comunicación dialogal. En el caso de los conocimientos sociales, Leman y Duveen (1996) han articulado los componentes disociados en el ME de la escisión: las identidades de género "restringían" la elaboración de ideas morales, ya que proveían del código para manejar las interacciones entre los sujetos ante una cuestión moral y le daban una dirección a la argumentación individual de la situación. Incluso, en la elaboración de aquellas identidades hay una influencia del desarrollo de las capacidades individuales (Lloyd, Duven, 1992).

Ahora bien, nuestro problema es poner de relieve que la propia psicología del desarrollo de los conocimientos sociales puede insertarse en un ME relacional, lo que permitiría establecer un diálogo con la TRS u otorgar un lugar a las RS en sus explicaciones, en contra de la tesis de la incompatibilidad o la inconmensurabilidad.

La tradición de investigación piagetiana ha sido interpretada "literalmente" por los psicólogos que han investigado los conocimientos sociales de modo descriptivo y desconociendo sus condiciones sociales. Por el contrario, se puede defender un constructivismo crítico orientado por un ME dialéctico, que vincula constitutivamente el conocimiento individual y sus condiciones sociales, retomando la herencia de Piaget en el Juicio Moral ( 1997), de los Estudios Sociológicos (1995), y de la más reciente Psicogénesis e Historia de la Ciencia (escrito con R. García, 1982), recuperando aspectos centrales de su núcleo y procediendo a ciertas revisiones.

Hemos caracterizado (Castorina, Faigenbaum, 2002; Lenzi, Castorina, 2000) la especificidad del "dominio social" por las interacciones no solo epistémicas sino sociales entre el sujeto y el objeto; hemos formulado la tesis de una "tensión" constitutiva entre la construcción individual de los conceptos (de gobierno, de autoridad escolar, de sanciones y castigos escolares, entre otros) y las prácticas sociales (sobre todo cuándo el sistemas de prácticas disciplinarias y normativas intervienen sobre la actividad cognitiva). Los conceptos infantiles 
se elaboran modulados por las prácticas sociales que son indisociables de la transmisión de creencias sociales que las expresan y a las que se dirigen. A diferencia del constructivismo "literal", nuestro enfoque de una dialéctica entre las condiciones sociales y la actividad individual es consistente con las teorías de la acción social que postulan un agente social activo bajo la presión social (Bourdieu, 1997).

Diversos trabajos sobre conceptos sociales (Helman, Castorina, 2005; Barreiro, Castorina, 2006; Lenzi, Castorina, 2000) sugieren - solo sugieren, no lo establecen por un estudio metodológico preciso - que las RS (e incluso su horizonte ideológico) están presentes en la construcción conceptual. Hay indicadores en las respuestas de los niños y alumnos de la vigencia de conocimientos ligados a la vida familiar, al anclaje en la memoria social, a fenómenos de adhesión y rechazo afectivos a figuras políticas, a creencias de conjunto sobre una sociedad naturalizada, que no son construcciones puramente individuales. Por ejemplo, las creencias de que la maestra es "la segunda mamá", que el presidente o la maestra son "benefactores", la ya mencionada "eternidad de la nación”, el carácter fuertemente moralista y personalizador del presidente y de la interpretación de la historia. Recientemente, se ha mostrado que la justicia inmanente estudiada por Piaget sería el resultado de la elaboración intelectual de un saber preexistente en la cultura, la ideología de la creencia en el mundo justo (Barreiro, 2007).

En general, encontramos una "polifasia" en el conocimiento político o histórico, constituido por conceptos e hipótesis infantiles, pasando por informaciones escolares, hasta aquellas creencias de la vida cotidiana y las ideologías. Una convivencia, a veces conflictiva, entre diferentes clases de conocimiento, sean las RS y los saberes conceptuales, así como diversas racionalidades. También entre las RS y las representaciones individuales, definidas por la diferenciación de significantes y significados, en un sentido constructivista.

Más aún, nuestra hipótesis central es que las RS no solo conviven con la construcción individual de conceptos - de autoridad política y escolar, nación, democracia representativa - sino que "restringen" a esta última. Es decir, constituyen (las RS o su horizonte ideológico) un saber social del que no pueden prescindir los niños y alumnos en su construcción conceptual. De este modo, estamos lejos de la exterioridad de la cultura que "estimula o frena" la elaboración individual. Las RS son parte de la construcción conceptual de cada 
individuo, en la medida que son las condiciones que posibilitan el conocimiento (son una materia prima de la elaboración intelectual) y limitan lo que se puede pensar autónomamente (por ejemplo, la versión benefactora del presidente dificulta la construcción conceptual del sistema político)

Pero es crucial para nuestra posición de psicólogos del desarrollo no considerar a las RS como una mera materia prima para la elaboración "cognitiva" de un sujeto puramente epistémico. Aunque una perspectiva constructivista no se ocupa da la constitución de la subjetividad social, es imprescindible contar con esta última, reconocer en la actividad de elaboración conceptual al sujeto comprometido con su identidad social, vinculada a las RS (Lloyd, Duveen, 2003). Debemos abrir la teoría psicológica a la imbricación del sujeto social en la actividad constructiva, la que además es consistente con nuestra tesis de la interacción social del sujeto y el objeto para los conocimientos sociales (en los intercambios reglados, en las interacciones con los dispositivos institucionales, en la apropiación de las creencias colectivas).

\section{UNA NOTA FINAL}

Hay que concluir que si bien el tema del desarrollo es común a los dos programas, el de las TRS y el constructivismo crítico (Duveen, 1994; Castorina, 2005) sus preguntas y su metodología de investigación son claramente diferentes. Sin embargo, según nuestra postulación, las hipótesis de un programa no implican la negación de las que corresponden al otro. Esta compatibilidad se basa en un marco epistémico relacional, que involucra la interacción dialéctica entre individuo y sociedad, así como una construcción del sujeto (individual o social) y del objeto (Castorina, Clemente, Barreiro, 2005). La diferencia de enfoques no conduce a incompatibilidad o a la creación de espacios epistemológicos divergentes, como se ha afirmado (Wagner et al., 1999). Lo que tenemos son focalizaciones en la investigación empírica en cada programa, respecto de determinadas temáticas, desde problemáticas diferentes y con la peculiaridad del recorte de los problemas y los procedimientos metodológicos.

Un corolario de lo dicho: hay elementos de juicio para sostener desde la TRS la tesis de que las creencias se construyen históricamente y se imponen a la consciencia individual, aunque sin determinarla unívocamente. De este modo, el estudio de las creencias sociales rechaza cualquier secuencia lineal y universal 
de desarrollo. Con cierta audacia, se puede sugerir que se va abriendo paso una interpretación del conocimiento social aproximado al proyecto discontinuista de la "psicología histórica" de Meyerson (1948).

De ahí que un programa constructivista de la psicología del desarrollo ganaría en utilizar el concepto de RS: le permitiría abandonar la exclusividad del sujeto epistémico, reconociendo explícitamente al sujeto de la identidad social, y haría factible situar a las ideas de los niños en un marco histórico, debido a la naturaleza sociogenética de las RS. Se puede afirmar, entonces, la actividad constructiva individual de las ideas sociales sin buscar una secuencia universal de ideas sobre el mundo social, un orden descontextuado de estructuraciones del mundo social.

Nuestros estudios psicogenéticos sobre la adquisición de conocimientos sociales dejan un lugar para las creencias sociales vinculadas a la memoria colectiva y a la historia que las configura. Estas intervienen, modulando la construcción individual de las ideas sociales. Gracias a esta contribución podríamos también recuperar un genuino constructivismo "situado" que admite el carácter histórico de sus condiciones sociales.

\section{BIBLIOGRAFÍA}

BARREIRO, A. La Justicia inmanente y la creencia en el mundo justo: relaciones entre la construcción individual del conocimiento y las creencias sociales. Buenos Aires, 2007. Facultad de Psicología, Universidad de Buenos Aires.

BARREIRO, A.; CASTORINA, J. A. Relaciones entre la noción de justicia y la creencia colectiva en el orden del mundo: una introducción a las creencias infantiles. Anuario de Investigaciones, Buenos Aires, v. 14, p. 167-176, 2006.

BOURDIEU, P Méditations pascaliennes. Paris: Seuil, 1997.

BRUNER, J. Celebrating the divergence: Piaget and Vygotsky. Human Development, Chicago, n.40, v.2, p.63-73, 1997.

CARRETERO, M.; KRIGER, M. La Usina de la patria y la mente de los alumnos: un estudio sobre las representaciones de las efemérides escolares argentinas. In: CARRETERO, M.; ROSA, A.; GONZÁLEZ, M. F. Enseñanza de la historia y memoria colectiva. Buenos Aires: Paidós, 2006, p. 161-190.

CASTORINA, J. A. El Impacto de la filosofía de la escisión en la psicología del desarrollo. Psykhe, v. I I, n. I, p. I5-27, 2002. 
La Investigación psicológica de los conocimientos sociales: los desafíos a la tradición constructivista. In: CASTORINA, J. A. (org.). Construcción conceptual y representaciones sociales. Buenos Aires: Miño y Dávila, 2005. Iª parte, cap. I.

CASTORINA, J. A; BARREIRO, A.; CLEMENTE, F. La Impronta del pensamiento piagetiano en la teoría de las representaciones sociales. In: CASTORINA, J. A. (org.). Construcción conceptual y representaciones sociales. Buenos Aires: Miño y Dávila, 2005. 3ª parte, cap.6.

CASTORINA, J. A; CLEMENTE, F; BARREIRO, A. El Conocimiento de los niños sobre la sociedad según el constructivismo y la teoría de las representaciones sociales. In: CASTORINA, J. A. (org.). Construcción conceptual y representaciones sociales. Buenos Aires: Miño y Dávila, 2005. 3ª parte, cap.7.

CASTORINA, J.A.; FAIGENBAUM, G. The Epistemological meaning constraints in the development of domain knowledge. Theory and Psychology, Londres, v. I2, n.3, p.3 I 5-334, 2002.

CASTORINA, J. A. et al. La Cultura en la psicología del desarrollo: una revisión crítica. In: CASTORINA, J. A. y cols. Cultura y conocimientos sociales: desafíos a la psicología del desarrollo. Buenos Aires: Aiqué, 2007.

CHARTIER, R. El Mundo como representación. Barcelona: Gedisa, 1995.

DELVAL, J. El Desarrollo humano. Madrid: Siglo Veintiuno, 1994.

DUVEEN, G. Crianças enquanto atores sociais: as representações sociais em desenvolvimento. In: GUARESCHI, P.; JOVCELOVITCH, S. (orgs.). Textos em representações sociais. Petrópolis: Vozes, 1994. p.26I-297.

The Child's re-construction of economics. In: BERENTZEN, S. (ed.). Ethnographic approaches to children's worlds and peer cultures. Trondhein: The Norwegian Centre for Child Research, 1989.

Introduction: the power of ideas. In: MOSCOVICI, S. Social representations: explorations in social psychology. New York: New York University Press, 200 I . p. I - 17.

The Psychosocial production of ideas: social representations and psychologic. Culture \& Psychology, v.4, n.4, p.455-476, 1998.

DUVEEN, G.; DE LA ROSA, A. M. Social representations and the genesis of social knowledge. Ongoing Production on Social Representations: Productions Vives sur les Representations Sociales, v. I, n.2/3, p.94-108, 1992.

ELIAS, N. Compromiso y distanciamiento. Barcelona: Península, 1989.

EMLER, N.; OHANA, J.; DICKINSON, J. Las Representaciones infantiles de las relaciones sociales. In: CASTORINA, J. A. Las Representaciones sociales. Barcelona: Gedisa, 2003. p.65-89. 
EMLER, N.; OHANA, J.; MOSCOVICI, S. Children's beliefs about institution roles: a crossnational study of representations of the teacher's role. British Journal of Educational Psychology, n.57, p.120-135, 1987.

GRIZE, J. B. Logique naturelle et representations sociales. In : JODELET, D. Les Répresentations sociales. Paris: PUF, 1986. p. I52-168.

HELMAN, M.; CASTORINA, J. A. La Institución escolar y las ideas de los niños sobre sus derechos. Revista del Instituto de Investigaciones en Ciencias de la Educación, Buenos Aires, v. 13, n.23, p.39-57, 2005.

HORN, A.; CASTORINA, J. A. Las Ideas de los niños sobre su derecho a la intimidad: construcción conceptual y restricciones institucionales. In: MEMORIAS de las Jornadas de Investigación, 2. 2007, Buenos Aires: Facultad de Psicología, Universidad Nacional de La Plata, 2007.

JAHODA, G. Critical notes and reflections on "social representations". European Journal of Social Psychology, v. I8, n.3, p.195-209, 1988.

JODELET, D. La representación social: fenómenos, concepto y teoría. In: MOSCOVICI, S. (comp.). Psicologia social, 2. Barcelona: Paidós, 1996. p.470-494.

JOVCHELOVITCH, S. Knowledge in context. London: Routledge, 2006.

LAUTIER, N. À la rencontre de l'histoire. Villeneuve d'Ascq: Presses Universitaires du Septembrion, 1997.

. L'Histoire en situation didactique: une pluralité des registres de savoir. In: HAAS, V. Les Savoirs du quotidien. Rennes. Presses Universitaires de Rennes, 2006. p.77-90.

LEMAN, P.; DUVEEN, G. Developmental differences in children's understanding of epistemic authority. European Journal of Social Psychology, v.26, n.5, p.45-59, 1996.

LENZI, A.; CASTORINA, J. A. Algunas reflexiones sobre una investigación psicogenética en conocimientos sociales: la noción de autoridad escolar. In: CASTORINA, J. A.; LENZI, A. (compls.). La Formación de los conocimientos sociales en los niños. Barcelona: Gedisa, 2000. p. $122-135$.

LLOYD, B.; DUVEEN, G. Gender identities and education: the imapact of starting scholl. London: Harvester- Wheatsheaf, 1992.

Un análisis semiótica del desarrollo de las representaciones de género. In:

CASTORINA, J. A. (comp.). Representaciones sociales. Barcelona: Gedisa, 2003. p.4I-64.

MACHADO, A; LOURENÇO, O; SILVA, F. Facts, concepts and theories: the shape of psychology's epistemic triangle. Behavior and Philosophy, n.28, p. I-40, 2000. 
MARKOVA, I. Dialogicality and social representations. Cambridge: Cambridge University Press, 2003.

MEYERSON, I. Les Functiones psychologiques et les oeuvres. Paris: Vrin, 1948.

MOSCOVICI, S. La Conciencia social y su historia. In: CASTORINA, J. A. (comp.). Representaciones sociales. Barcelona: Gedisa, 2003. p.91-1 I0.

The Phenomenon of social representation. In: FAR, R. M.; MOSCOVICl, S. (compls.). Social representations. Cambridge: Cambridge University Press, 1984. p.3-69.

Notas towards a description of social representations. European Journal of Social Psychology, v. 18, n.3, p.21 1-250, 1988.

(org.). Psicología Social, 2. Barcelona: Paidós, 1986.

OVERTON, W. Developmental psychology: philosophy, concepts and methodology. In: DAMON, W.; LERNER, R. M. (eds.). Handbook of child psychology, I. 5. ed. New Cork: Wiley, 1998. p. 107-188.

PIAGET, J. El Criterio moral en el niño. Barcelona: Fontanella, 1997.

El Juicio y el razonamiento en el niño. Madrid: Ediciones de la Lectura, 1940. Sociological studies. New York: Routledge, 1995.

PIAGET, J.; GARCÍA, R. Psicogénesis e historia de la ciencia. México: Siglo Veintiuno, 1982.

POTTER, J.; EDWARDS, D. Social representations and discursive psychology: from cognition to action. Culture \& Psychology, v.5, n.4, p.447-458, 1999.

SMEDSLUND, J. Social representations and psychologic. Culture \& Psychology, v.4, n.4, p.435-454, 1998.

TAYLOR, C. Philosophical arguments. Cambridge: Harvard University Press, 1995.

VAN DER VEER, R. The Concept of culture in Vigotsky's thinking. Culture and Psychology, v.2. n.3, p.247-267, 1996.

WAGNER, W. Social cognition vs. social representations : a comment on Duveen \& de Rosa. Ongoing Production on Social Representations: Productions Vives sur les Répresentations Sociales, v. I, n.2/3, p. 109-। 15, 1992.

WAGNER, W. et al. Theory and method of social representations. Asian Journal of Social Psychology, n.2, p.95-125, 1999.

Recebido em: dezembro 2007

Aprovado para publicação em: março 2008 